\title{
SYNTHETIC STUDIES ON 3-ARYLOXYETHYL-4-ARYLIDENE AMINO-5-MERCAPTO-1,2,4-TRIAZOLES AND BIOLOGICAL EVALUATION AS ANTIFUNGAL AND ANTIBACTERIAL AGENTS
}

\author{
K. Sujatha ${ }^{1}$, Balakrishna Kalluraya ${ }^{2}$ and Shrinivas D. Joshi ${ }^{3}$ \\ ${ }^{1}$ Department of Studies in Chemistry, Karnatak University, Dharwad-580003Karnataka, India \\ ${ }^{2}$ Department of Studies in Chemistry, Mangalore University, i-574199 Karnataka, India \\ ${ }^{3}$ Department of Pharmaceutical Chemistry, S.E.T's College of Pharmacy, Dharwad \\ *E-mail: sujathakorganic@gmail.com
}

\begin{abstract}
A novel series of 3-aryloxyethyl-4-arylidene amino-5-mercapto-1,2,4-triazoles were prepared from 5-( $\alpha$-aryloxy ethyl)-1,2,4 triazole, The structures of new compounds have been established by spectral and analytical data. The newly synthesized compounds have been tested for their antimicrobial activity.
\end{abstract}

Keywords: Triazole, Schiff's Base, Docking Study.

(C) RASĀYAN. All rights reserved

\section{INTRODUCTION}

Azoles are the most widely used antifungal agents in current clinical use. ${ }^{1}$ Due to the increased incidence of invasive fungal infections worldwide in the past two decades. ${ }^{2,3}$ IFIs increased the high rate of morbidity and mortality and to diagnose, treat and prevent is the most difficult task. Candida spp. is the fourth most common nosocomial pathogen causing the highest crude mortality rate (40\%) in USA. ${ }^{4}$ In patients with hematological malignancies ${ }^{5}$ apart from Aspergillus spp., new and emerging fungal pathogens like Zygomycetes, Fusarium spp. Or Scedosporium spp. have become major pathogens resulting in mortality rate is $\geq 70 \%$. Their susceptibility to a currently available antifungal is limited.

The Standard systemic antibiotic therapy is unsatisfactory in certain circumstances. More attention has to be focused on solving the problem of multidrug-resistant bacteria and the staggering costs and consequences resulting from this. The resistance developed by microorganisms for many antimicrobial agents compels to search for new synthetic antimicrobial substances.

Triazole moiety can be considered as a bioisostere of imidazole, which imparts the main functional role in the azole group of antifungal drugs (i.e., fluconazole). The biological activities of various triazole derivatives have been extensively studied. Even though triazole and imidazole are five-membered ring heterocycles, triazoles contain three-ring nitrogen atoms, and imidazole has two. On comparison of triazoles with imidazoles (clotrimazole, ketoconazole, miconazole), triazoles exhibit greater target specificity, increased potency and an expanded spectrum of activity and moreover are less susceptible to metabolic degradation. ${ }^{6,7}$

Triazole has versatility infusing to various ring systems and possesses a broad spectrum of biological activity. Since triazole is capable of forming more hydrogen bond, which is the most essential aspect in binding biomolecular targets as well as to increase the solubility. ${ }^{8}$ Moreover, to have an innovative bifunctional drug, triazoles can function as attractive linker units which could connect two pharmacophores, and thus plays a vital role in constructing bioactive and functional molecules. ${ }^{9-11}$ significantly expanding the chemical space of triazole scaffolds possessing potent activities or enhancing biological activities. ${ }^{12}$ Additionally, extensive studies have shown that the addition of alkyl chains and/or

Rasayan J. Chem., 12(3), 1405-1417(2019)

http://dx.doi.org/10.31788/RJC.2019.1235200

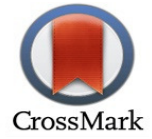


various aromatic substituents, especially containing halogen atoms, has a major impact on the antimicrobial activities Itraconazole ${ }^{13-16}$, voriconazole ${ }^{17}$, Ravuconazole ${ }^{18}$ are major antifungal agents containing halogens presently available in the drug market for the treatment of fungal infections. Among the most important effects, triazole derivatives have been reported to exhibit antibacterial ${ }^{20-34}$ antifungal ${ }^{8,20-23,35-37}$ and antimycobacterial ${ }^{9-12}$, properties. These observations motivate for the continuation of our search for new bioactive molecules.

\section{EXPERIMENTAL}

\section{Materials and Methods}

${ }^{1} \mathrm{H}$ NMR spectra were recorded on a Bruker WH-200 (400 MHz) spectrometer in $\mathrm{CDCl}_{3}$ or DMSO-d $\mathrm{d}_{6}$ as a solvent, using TMS as an internal standard and chemical shifts are expressed in $\delta$ scale. The melting points of all the compounds were determined by the open capillary method. IR spectra of all the newly synthesized compounds were obtained in $\mathrm{KBr}$ disc on a Shimadzu FT-IR 157 spectrophotometer. Mass spectra were determined on a Jeol SX 102/Da-600 mass spectrometer/ Data System using Argon/Xenon $\left(6 \mathrm{kv}, 10 \mathrm{~mA}^{\circ}\right)$ as the FAB gas. The accelerating voltage was $10 \mathrm{kV}$ and spectra are recorded at room temperature. The formation of products was monitored by TLC.

\section{General Procedure for the Synthesis of Methyl aryloxypropanoate (1)}

A mixture of substituted phenol $(0.2 \mathrm{~mol})$, methyl 2-chloropropanoate $(0.2 \mathrm{~mol})$ and anhydrous potassium carbonate $(41.4 \mathrm{~g}, 0.3 \mathrm{~mol})$ in dry acetone $(500 \mathrm{ml})$ was refluxed on a water bath for about 16 hours. The reaction mixture was filtered hot to remove insoluble and the reaction mass was concentrated under reduced pressure, As such the crude methyl aryloxypropanoate ester was taken for the next stage without any further purification.

\section{General Procedure for the Synthesis of Methyl aryloxypropanohydrazide (2)}

A mixture of crude methyl aryloxypropanoate $(0.1 \mathrm{~mol})$ and $5 \mathrm{ml}$ of $99 \%$ hydrazine hydrate $(0.1 \mathrm{~mol})$ in $40 \mathrm{ml}$ of ethanol was refluxed on a water bath for eight hours, the reaction mixture on cooling gave crude mass of aryloxypropanohydrazide which was collected by filtration and recrystallized from alcohol to get white crystals in good yield $(86 \%)$.

\section{General Procedure for the Preparation of Aryloxypropano potassium dithiocarbazinates (3)}

A mixture of substituted aryloxypropanohydrazide $(0.1 \mathrm{~mol})$ in ethanol $(100 \mathrm{ml})$ was added slowly to a solution of potassium hydroxide $(0.15 \mathrm{~mol})$ in ethanol $(50 \mathrm{ml})$. The resulting mixture was stirred well till a clear solution was obtained. Carbon disulphide $(0.15 \mathrm{~mol})$ was added drop wise to it and stirred vigorously. The temperature was not allowed to rise above $30^{\circ} \mathrm{c}$. Solid mass began to separate immediately. It was further stirred for 24 hours at room temperature. The resulting mixture was diluted with ether $(100 \mathrm{ml})$ and the precipitate formed was collected by filtration, washed with dry ether and dried at $65^{\circ} \mathrm{c}$ under vacuum. The salt obtained by this procedure was used for the next reaction without further purification.

General Procedure for the Preparation of Substituted 4-amino-5-mercapto-1,2,4-triazoles (4a-d), (5e)

Potassium dithiocarbazinate $(0.1 \mathrm{~mol})$ and hydrazine hydrate $(99 \%, 0.2$ mole) and $2 \mathrm{ml}$ water were gently heated so that it started to boil in about thirty minutes, The reaction mass diluted with water, and neutralized with hydrochloric acid. The product separated was filtered, dried and recrystallized from ethanol

General Procedure for the Preparation of 3-aryloxyethyl-4-arylidene amino-5-mercapto-1,2,4triazoles (6a-o)

Substituted 4-amino-5-mercapto-1,2,4-triazoles ( 0.1 mole), substituted aldehydes ( $0.1 \mathrm{~mole})$, 5ml of isopropyl alcohol and a drop of con. Sulphuric acid was refluxed for an hour on a water bath. Thus obtained product filtered and recrystallized from ethanol 
RASĀYAN J. Chem.

Vol. 12 | No. 3 |1405 - 1417| July - September | 2019

The structures of the newly synthesized triazoles (4a-d), (5e) and their Schiff bases were confirmed on the basis of analytical and spectral data. The characterization data of these compounds are given in Table- 1 and 2 respectively.

Table-1: Characterization Data of 3-(1-arylethyl) / (1-imidazolylethyl) -4-arylidene amino-5-mercapto-1,2,4triazoles $(6 \mathrm{a}-\mathrm{o})$

\begin{tabular}{|c|c|c|c|c|c|c|c|}
\hline \multirow[t]{2}{*}{$\begin{array}{l}\text { Compd. } \\
\text { No }\end{array}$} & \multirow[t]{2}{*}{$\mathrm{Ar}$} & \multirow[t]{2}{*}{$\mathrm{R}$} & \multirow[t]{2}{*}{$\begin{array}{l}\text { Yield }(\%) \\
\text { M.P }\left({ }^{\circ} \mathrm{C}\right)\end{array}$} & \multirow{2}{*}{$\begin{array}{c}\text { Colour and } \\
\text { Crystal } \\
\text { form }\end{array}$} & \multicolumn{3}{|c|}{$\begin{array}{l}\text { Elemental analysis } \\
\text { (Calc) Found }\end{array}$} \\
\hline & & & & & $\mathrm{C}$ & $\mathrm{H}$ & $\mathrm{N}$ \\
\hline 6a & Phenoxy & Biphenyl & $\begin{array}{c}55 \\
170-72\end{array}$ & $\begin{array}{l}\text { Light } \\
\text { yellow } \\
\text { crystals }\end{array}$ & $\begin{array}{c}68.98 \\
(68.99)\end{array}$ & $\begin{array}{l}5.03 \\
(5.04)\end{array}$ & $\begin{array}{c}13.99 \\
(13.98)\end{array}$ \\
\hline 6b & Phenoxy & 4-Chlorophenyl & $\begin{array}{c}58 \\
142 \\
\end{array}$ & $\begin{array}{l}\text { Yellow } \\
\text { crystals }\end{array}$ & $\begin{array}{c}56.90 \\
(56.89) \\
\end{array}$ & $\begin{array}{r}4.25 \\
(4.21) \\
\end{array}$ & $\begin{array}{c}15.61 \\
(15.59) \\
\end{array}$ \\
\hline 6c & Phenoxy & Pyridyl & $\begin{array}{c}61 \\
148-150\end{array}$ & $\begin{array}{c}\text { Pale } \\
\text { yellow } \\
\text { needles }\end{array}$ & $\begin{array}{l}59.06 \\
(59.04)\end{array}$ & $\begin{array}{c}4.65 \\
(4.66)\end{array}$ & $\begin{array}{c}21.52 \\
(21.53)\end{array}$ \\
\hline 6d & 4-Chlorophenoxy & Biphenyl & $\begin{array}{c}54 \\
106-08 \\
\end{array}$ & $\begin{array}{l}\text { Yellow } \\
\text { needles }\end{array}$ & $\begin{array}{c}63.51 \\
(63.50) \\
\end{array}$ & $\begin{array}{c}4.40 \\
(4.39) \\
\end{array}$ & $\begin{array}{c}12.88 \\
(12.89) \\
\end{array}$ \\
\hline $6 e$ & 4-Chlorophenoxy & 4-Chlorophenyl & $\begin{array}{c}61 \\
155-156\end{array}$ & $\begin{array}{c}\text { Pale } \\
\text { yellow } \\
\text { micro } \\
\text { needles }\end{array}$ & $\begin{array}{c}51.92 \\
(51.93)\end{array}$ & $\begin{array}{c}3.59 \\
(3.58)\end{array}$ & $\begin{array}{l}14.25 \\
(14.29)\end{array}$ \\
\hline $6 f$ & 4-Chlorophenoxy & Pyridyl & $\begin{array}{c}53 \\
256\end{array}$ & $\begin{array}{c}\text { Pale } \\
\text { yellow } \\
\text { crystals }\end{array}$ & $\begin{array}{c}53.41 \\
(53.42)\end{array}$ & $\begin{array}{c}3.92 \\
(3.91)\end{array}$ & $\begin{array}{l}19.46 \\
(19.45)\end{array}$ \\
\hline $6 \mathrm{~g}$ & $\begin{array}{c}2,4- \\
\text { Dichlorophenoxy }\end{array}$ & Biphenyl & $\begin{array}{c}55 \\
204\end{array}$ & $\begin{array}{c}\text { Light } \\
\text { yellow } \\
\text { crystals }\end{array}$ & $\begin{array}{l}58.85 \\
(58.84)\end{array}$ & $\begin{array}{c}3.87 \\
(3.86)\end{array}$ & $\begin{array}{l}11.94 \\
(11.93)\end{array}$ \\
\hline $6 \mathbf{h}$ & $\begin{array}{c}2,4- \\
\text { Dichlorophenoxy }\end{array}$ & 4-Chlorophenyl & $\begin{array}{c}64 \\
160 \\
\end{array}$ & $\begin{array}{l}\text { Yellow } \\
\text { crystals }\end{array}$ & $\begin{array}{c}47.74 \\
(47.75) \\
\end{array}$ & $\begin{array}{c}3.06 \\
(3.05) \\
\end{array}$ & $\begin{array}{c}13.10 \\
(13.11) \\
\end{array}$ \\
\hline $6 \mathbf{i}$ & $\begin{array}{c}2,4- \\
\text { Dichlorophenoxy }\end{array}$ & Pyridyl & $\begin{array}{c}54 \\
289 \\
\end{array}$ & $\begin{array}{l}\text { Cream } \\
\text { needles }\end{array}$ & $\begin{array}{c}48.74 \\
(48.73) \\
\end{array}$ & $\begin{array}{c}3.32 \\
(3.33) \\
\end{array}$ & $\begin{array}{c}17.76 \\
(17.75) \\
\end{array}$ \\
\hline $6 \mathbf{j}$ & $\begin{array}{c}2,6- \\
\text { Dimethylphenoxy }\end{array}$ & Biphenyl & $\begin{array}{c}59 \\
166-68\end{array}$ & $\begin{array}{c}\text { Pale } \\
\text { yellow } \\
\text { crystals }\end{array}$ & $\begin{array}{l}70.07 \\
(70.08)\end{array}$ & $\begin{array}{c}5.64 \\
(5.66)\end{array}$ & $\begin{array}{c}13.07 \\
(13.05)\end{array}$ \\
\hline 6k & $\begin{array}{c}2,6- \\
\text { Dimethylphenoxy }\end{array}$ & 4-Chlorophenyl & $\begin{array}{c}57 \\
160 \\
\end{array}$ & $\begin{array}{l}\text { Yellow } \\
\text { crystals }\end{array}$ & $\begin{array}{c}58.98 \\
(58.99) \\
\end{array}$ & $\begin{array}{c}4.95 \\
(4.96) \\
\end{array}$ & $\begin{array}{c}14.48 \\
(14,49) \\
\end{array}$ \\
\hline 61 & $\begin{array}{c}2,6- \\
\text { Dimethylphenoxy }\end{array}$ & Pyridyl & $\begin{array}{c}57 \\
289\end{array}$ & $\begin{array}{l}\text { Yellow } \\
\text { needles }\end{array}$ & $\begin{array}{c}61.17 \\
(61.18) \\
\end{array}$ & $\begin{array}{c}5.42 \\
(5.43) \\
\end{array}$ & $\begin{array}{c}19.81 \\
(19.82)\end{array}$ \\
\hline $6 \mathrm{~m}$ & Imidazolyl & Biphenyl & $\begin{array}{c}46 \\
135 \\
\end{array}$ & $\begin{array}{l}\text { Yellow } \\
\text { crystals }\end{array}$ & $\begin{array}{c}61.52 \\
(61.53) \\
\end{array}$ & $\begin{array}{c}4.65 \\
(4.66) \\
\end{array}$ & $\begin{array}{c}21.52 \\
(21.53) \\
\end{array}$ \\
\hline 6n & Imidazolyl & 4-Chlorophenyl & $\begin{array}{c}54 \\
157\end{array}$ & $\begin{array}{c}\text { Creamish } \\
\text { yellow } \\
\text { needles } \\
\end{array}$ & $\begin{array}{c}48.51 \\
(48.52)\end{array}$ & $\begin{array}{c}3.76 \\
(3.77)\end{array}$ & $\begin{array}{c}24.09 \\
(24.10)\end{array}$ \\
\hline 60 & Imidazolyl & Pyridyl & $\begin{array}{c}45 \\
159\end{array}$ & $\begin{array}{c}\text { Light } \\
\text { brown } \\
\text { crystals }\end{array}$ & $\begin{array}{c}49.51 \\
(49.53)\end{array}$ & $\begin{array}{c}4.16 \\
(4.17)\end{array}$ & $\begin{array}{c}31.09 \\
(31.10)\end{array}$ \\
\hline
\end{tabular}

\section{Spectral Data of Triazole}

4-amino-5-[1-(2,4-dichlorophenoxy)ethyl]-4H-1,2,4-triazole-3-thiol (4d)

IR: $\left(\mathrm{KBr}:: \gamma / \mathrm{cm}^{-1}\right)$ : 3300-3400 $\left(\mathrm{NH}_{2}\right), 1130-1180(\mathrm{C}=\mathrm{S}$ stretching $) .{ }^{1} \mathrm{H}-\mathrm{NMR}\left(\delta \mathrm{ppm}, \mathrm{DMSO}-\mathrm{d}_{6}, 400\right.$ $\mathrm{MHz}): 1.54\left(\mathrm{~d}, 3 \mathrm{H}, \mathrm{CH}_{3}\right), 4.4(\mathrm{q}, 1 \mathrm{H}, \mathrm{OCH}), 6.5-7.5$ (m, 3H, $\left.\mathrm{Ar}-\mathrm{H}\right), 8.9$ (br, $\left.2 \mathrm{H}, \mathrm{NH}_{2}\right)$ 
4-amino-5-[1-(4-chlorophenoxy)ethyl]-4H-1,2,4-triazole-3-thiol (4b)

${ }^{1} \mathrm{H}-\mathrm{NMR}\left(\delta \mathrm{ppm}, \mathrm{DMSO}-\mathrm{d}_{6}, 400 \mathrm{MHz}\right): \mathrm{d} 1.6\left(\mathrm{~d}, 3 \mathrm{H}, \mathrm{CH}_{3}\right), 4.6(\mathrm{q}, 1 \mathrm{H}, \mathrm{OCH}), 6.5-7.5(\mathrm{~m}, 3 \mathrm{H}, \mathrm{Ar}-\mathrm{H})$, 10.28. 8.9 (br, $2 \mathrm{H}, \mathrm{NH}_{2}$ ),

4-amino-5-[1-(2,6-dimethylphenoxy)ethyl]-4H-1,2,4-triazole-3-thiol (4c)

${ }^{1} \mathrm{H}$ NMR $(\delta$ ppm, DMSO-d 6 , $400 \mathrm{MHz}): \delta, 1.4\left(\mathrm{~d}, 3 \mathrm{H}, \mathrm{CH}_{3}\right), 2.2(\mathrm{~s}, 6 \mathrm{H}, 2 \mathrm{X} \mathrm{CH}$ ), $4.4(\mathrm{q}, 1 \mathrm{H}, \mathrm{O}-\mathrm{CH}), 6.7-$ 7.5 (m, 3H, Ar-H), 10.1 (br, 2H, $\left.\mathrm{NH}_{2}\right)$.

Spectral Data of 3-Aryloxyethyl-4-arylidene amino-5-mercapto-1,2,4-triazoles 4-\{biphenyl-4-ylmethylene]amino\}-5-[1-(2,4-dichlorophenoxy)ethyl]-2,4-dihydro-3H-1,2,4-triazole3-thione (6g)

IR: $\left(\mathrm{KBr}:: \gamma / \mathrm{cm}^{-1}\right): 1619(\mathrm{C}=\mathrm{N}),{ }^{1} \mathrm{H}$ NMR $\left(\delta \mathrm{ppm}, \mathrm{DMSO}-\mathrm{d}_{6}, 400 \mathrm{MHz}\right): \delta, 1.49\left(\mathrm{~d}, 3 \mathrm{H}, \mathrm{CH}_{3}\right), 4.2(\mathrm{q}$, $1 \mathrm{H}, \mathrm{O}-\mathrm{CH})$, 7.4-7.5 (m, 12H, Ar-H), 8.47(s, 1H, N=CH).

\section{4-\{[biphenyl-4-ylmethylene]amino\}-5-[1-(1H-imidazol-1-yl)ethyl]-4H-1,2,4-triazole-3-thiol (6m)}

${ }^{1} \mathrm{H}$ NMR $\left(\delta \mathrm{ppm}, \mathrm{DMSO}_{-}, 400 \mathrm{MHz}\right): \delta, 1.9\left(\mathrm{~d}, 3 \mathrm{H}, \mathrm{CH}_{3}\right), 5.9(\mathrm{q}, 1 \mathrm{H}, \mathrm{NCH}), 7.01-8.01(\mathrm{~m}, 12 \mathrm{H}$, aromatic and imidazle protons) $9.95(\mathrm{~s}, 1 \mathrm{H}, \mathrm{N}=\mathrm{CH}), 14.12(\mathrm{~s}, 1 \mathrm{H}, \mathrm{SH})$.

\section{RESULTS AND DISCUSSION}

The four differently substituted-3-aryloxyethyl-4-amino-5-mercapto triazoles were employed in the preparation of corresponding Schiff"s bases. All the substituted triazoles were prepared to start from substituted phenols. Substituted phenols on reacting with methyl 2-chloropropanoate in dry acetone in the presence of anhydrous potassium carbonate gave $\alpha$-methylaryloxypropanoates (1). Hydrazinolysis of these esters with hydrazine hydrate in absolute ethanol yielded $\alpha$-aryloxypropano hydrazides (2). Potassium dithiocarbazinates (3) were prepared by reacting hydrazides (2) with carbon disulphide in absolute alcohol in the presence of base potassium hydroxide. Thus obtained potassium dithiocarbazinates (3) cyclization with hydrazine hydrate yielded 3-aryloxyethyl-4-amino-5-mercapto-1,2,4-triazoles (4a-d) The synthetic route followed for obtaining title compound is out lined in Scheme-1. Similarly 3-[1-(1Himidazol-1-yl)ethyl]-4H-1,2,4-triazol-4-amine (5e) was obtained by employing the same procedure (Scheme-1).

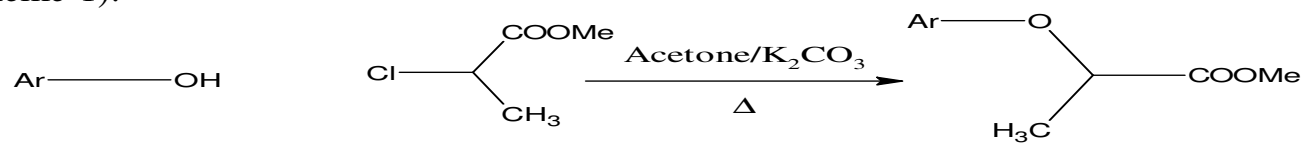

(1)

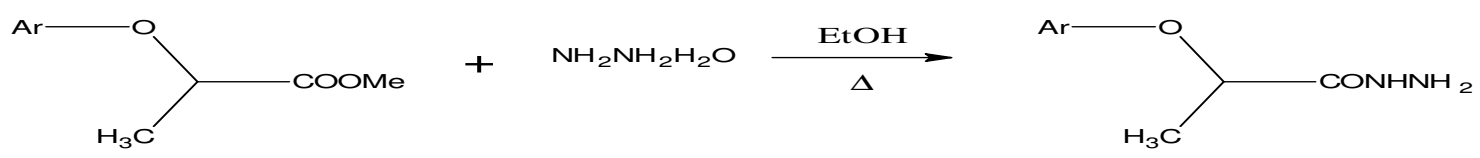

(2)
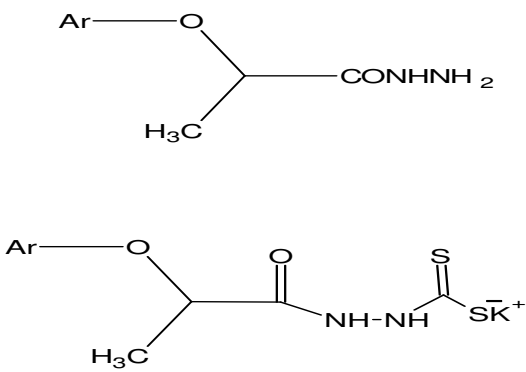

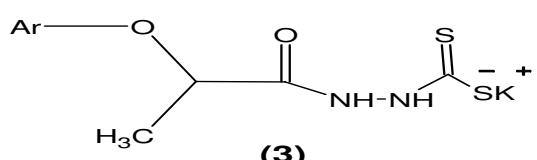

(3)

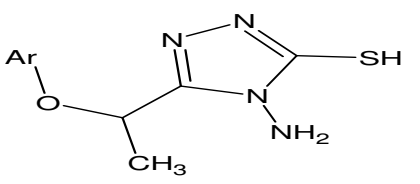

(4)

Scheme-1 


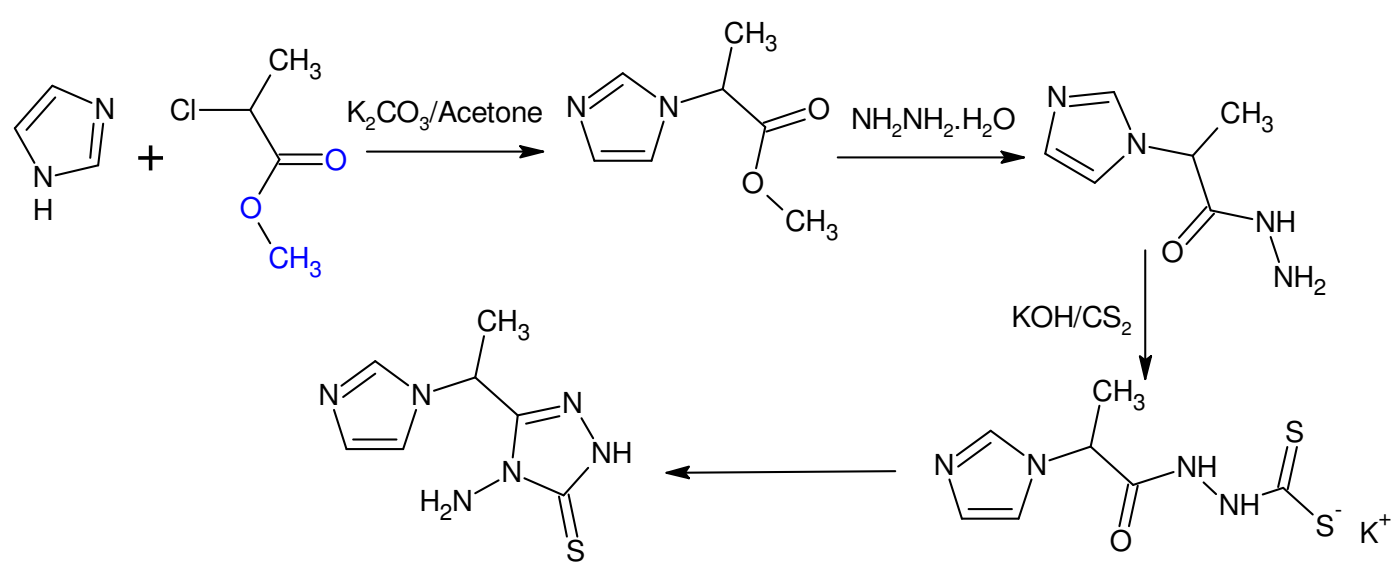

$(5 e)$

Scheme-2

Condensation of 4-amino-5-(1-aryloxyethyl)-4H-1,2,4-triazole-3-thioles (4a-d.) and 3-[1-(1H-imidazol1-yl)ethyl]-4H-1,2,4-triazol-4-amine (5e) with appropriately substituted aromatic aldehydes in ethanol as solvent employing con. Sulphuric acid as catalyst gave the novel series of 3-aryloxyethyl-4-arylidene amino-5-mercapto-1,2,4-triazoles, 3-[1-(1H-imidazol-1-yl)ethyl]-4-[arylmethylene]amino-4H-1,2,4triazole-5-thiol (Schiff's bases) (6a-o)(Scheme-3).<smiles>[R]C(C)c1nnc(S)n1N</smiles>

$$
\mathrm{R}=\text { Substituted aryloxy, imidazole }
$$

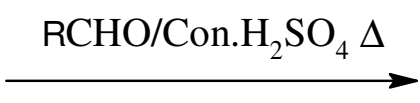

Scheme-3<smiles>[R]C=Nn1c(S)nnc1C([R])C</smiles>

(6)

\section{Antimicrobial Studies}

The newly synthesized 3-aryloxyethyl-4-arylidene amino-5-mercapto-1,2,4-triazoles were screened for their antibacterial activity invitro against fungus namely Candida albicans and the Gram-positive bacteria namely Escherichia coli, Staphylococcus aureus, and Gram-negative bacteria namely Pseudomonas aeruginosa, Bacillus subtitis by disc diffusion method. The test 3-aryloxyethyl-4-arylidene amino-5mercapto-1,2,4-triazoles were dissolved in N.N-dimethyl formamide (DMF) to obtain a solution of $10 \mu \mathrm{g} / \mathrm{ml}$ concentration. Penicillin and Flucanozole were used as reference standards to evaluate the potency of the newly synthesized Schiff bases The inhibition zones of microbial growth produced by different Schiff bases were measured at the end of an incubation period of 48 hours at $37^{\circ} \mathrm{C}$. DMF alone showed no inhibition zone. The results are shown in the Table-2.

The in vitro antimicrobial activity study revealed that few of the tested compounds showed significant antifungal activity. Compounds 6a, 6f ,69, 61,6m, showed good activity and 60 showed better against three different microbial strains ( bacteria) but less active compared to the standard. The structural makeup of the compounds was thought of to be responsible for their antimicrobial activities.

The compounds $6 \mathbf{6 a}, \mathbf{6 b}, \mathbf{6 c}, \mathbf{6 e}, \mathbf{6 f}, \mathbf{6 g} \mathbf{6 i}, \mathbf{6 k}, \mathbf{6}, \mathbf{6 m}$, showed very good activity against microbial fungal strains even better than the standard again the structural makeup of the compounds were thought of to be responsible for their antimicrobial activities. 
RASĀYAN J. Chem.

Vol. 12 | No. 3 |1405 - 1417| July - September | 2019

Table-2: Antibacterial and Antifungal Data of Schiff's Bases (6a-o).

\begin{tabular}{|c|c|c|c|c|c|}
\hline \multirow[t]{2}{*}{$\begin{array}{l}\text { Compd. } \\
\text { No }\end{array}$} & \multicolumn{4}{|c|}{ Antibacterial Activity (MIC in $\mu \mathrm{g} / \mathrm{mL}$ ) } & \multirow{2}{*}{$\begin{array}{c}\text { Antifungal } \\
\text { Activity (MIC } \\
\text { in } \mu \mathrm{g} / \mathrm{mL} \text { ) } \\
\text { C.albicans }\end{array}$} \\
\hline & E.coli & S.aureus & P.auriginosa & B.subtitis & \\
\hline $6 a$ & 6.25 & 6.25 & 3.12 & 3.12 & 3.12 \\
\hline $6 \mathrm{~b}$ & 6.25 & 6,25 & 3.12 & 6.25 & 3.12 \\
\hline $6 \mathrm{c}$ & 6.25 & 3.12 & 6.25 & 12.5 & 3.12 \\
\hline $6 \mathrm{~d}$ & 6.25 & 12.5 & 6.25 & 6.25 & 6.25 \\
\hline $6 e$ & 3.12 & 6.25 & 3.12 & 12.5 & 3.12 \\
\hline $6 f$ & 12.5 & 12.5 & 3.12 & 3.12 & 3.12 \\
\hline $6 g$ & 6.25 & 6.25 & 3.12 & 3.12 & 3.12 \\
\hline $6 \mathrm{~h}$ & 6.25 & 6.25 & 6.25 & 6.25 & 6.25 \\
\hline $6 \mathrm{i}$ & 6.25 & 12.5 & 3.12 & 12.5 & 3.12 \\
\hline $6 j$ & 12.5 & 6.25 & 6.25 & 3.12 & 3.12 \\
\hline $6 \mathrm{k}$ & 6.25 & 6.25 & 6.25 & 12.5 & 6.25 \\
\hline 61 & - & 6.25 & 3.12 & 3.12 & 3.12 \\
\hline $6 \mathrm{~m}$ & 6.25 & 3.12 & 6.25 & 3.12 & 3.12 \\
\hline $6 n$ & 3.12 & 6.25 & 3.12 & 6.25 & 6.25 \\
\hline 60 & 3.12 & 6.25 & 3.12 & 3.12 & 6.25 \\
\hline $\begin{array}{l}\text { Standard: } \\
\text { Pencillin }\end{array}$ & 0.12 & 0.12 & 0.12 & 0.12 & --- \\
\hline $\begin{array}{c}\text { Standard: } \\
\text { Flucanozole }\end{array}$ & - & - & - & - & 8.0 \\
\hline $\begin{array}{c}\text { Control: } \\
\text { DMF }\end{array}$ & - & - & ---- & --- & --- \\
\hline
\end{tabular}

\section{Docking Study}

The crystal structures of Candida albicans dihydrofolate reductase (PDB ID: 1AI9, X-Ray Diffraction, $1.85 \AA$ ) used for the docking study was obtained from the Protein Data Bank. The proteins were prepared for docking by adding polar hydrogen atom with Gasteiger-Huckel ${ }^{39,40}$ charges and water molecules were removed. The 3D structure for the ligands was generated with the help of SKETCH module implemented in the SYBYL program (Tripos Inc., St. Louis, USA) and its energy-minimized conformation was obtained with the help of the Tripos force field using Gasteiger-Huckel ${ }^{39}$ charges and molecular docking was performed with Surflex-Dock program that is interfaced with Sybyl-X 2.0. ${ }^{39}$ and other miscellaneous parameters were assigned with the default values given by the software (Table-3). Surflex Docking score ( $\mathrm{kcal} / \mathrm{mol})$ of the synthesized derivatives for Candida albicans (PDB ID: 1AI9)

Table-3: Surflex Docking Score (Kcal/mol) of the Synthesized Derivatives for Candida albicans (PDB ID: 1AI9)

\begin{tabular}{|c|c|c|c|c|c|c|c|}
\hline Compounds & C Score ${ }^{a}$ & $\begin{array}{l}\text { Crash } \\
\text { Score }^{b}\end{array}$ & $\begin{array}{l}\text { Polar } \\
\text { Score }^{c}\end{array}$ & D Score ${ }^{d}$ & $\begin{array}{l}\text { PMF } \\
\text { Score }^{\text {e }}\end{array}$ & G Score ${ }^{f}$ & $\begin{array}{l}\text { Chem } \\
\text { Score }^{\mathrm{g}}\end{array}$ \\
\hline Flucanazole & 6.12 & -2.43 & 4.76 & -25.709 & -38.035 & -201.77 & -11.08 \\
\hline $6 \mathrm{k}$ & 5.94 & -2.86 & 2.20 & -98.156 & -6.711 & -269.697 & -20.399 \\
\hline $6 a$ & 5.66 & -1.05 & 1.27 & -91.747 & -26.506 & -221.456 & -25.332 \\
\hline $6 f$ & 5.48 & -1.44 & 2.28 & -110.991 & -2.655 & -207.588 & -18.748 \\
\hline $6 d$ & 5.42 & -1.70 & 1.29 & -109.632 & -12.221 & -211.603 & -19.394 \\
\hline 61 & 5.41 & -3.37 & 0.61 & -81.826 & -13.065 & -250.471 & -21.098 \\
\hline $6 \mathrm{c}$ & 5.40 & -1.38 & 1.29 & -96.597 & -18.605 & -212.002 & -17.376 \\
\hline $6 \mathrm{e}$ & 5.28 & -1.97 & 2.48 & -88.919 & -7.787 & -233.752 & -18.824 \\
\hline $6 \mathrm{j}$ & 5.20 & -0.98 & 0.00 & -101.811 & -11.576 & -191.981 & -17.888 \\
\hline $6 \mathrm{~b}$ & 5.05 & -1.55 & 0.86 & -107.855 & 0.189 & -229.186 & -19.707 \\
\hline $6 n$ & 5.03 & -0.30 & 3.02 & -23.223 & -43.721 & -176.222 & -16.807 \\
\hline $6 \mathrm{i}$ & 4.88 & -1.34 & 3.06 & -110.494 & 35.103 & -176.978 & -23.125 \\
\hline
\end{tabular}


RASĀYAN J. Chem.

Vol. 12 | No. 3 |1405 - 1417| July - September | 2019

\begin{tabular}{c|l|l|l|l|l|l|l}
\hline $6 \mathrm{~m}$ & 4.82 & -3.58 & 2.10 & -106.972 & -7.159 & -263.751 & -22.379 \\
\hline $6 \mathrm{~g}$ & 4.73 & -0.79 & 1.55 & -89.748 & -19.844 & -195.147 & -23.929 \\
\hline $6 \mathrm{~h}$ & 4.41 & -1.10 & 3.70 & -41.811 & -34.650 & -154.839 & -16.512 \\
\hline $6 \mathrm{o}$ & 3.90 & -0.48 & 0.99 & -68.750 & -6.805 & -163.985 & -13.698 \\
\hline
\end{tabular}

\section{Docking Study of Triazole Derivatives}

The docking study revealed that all the compounds have shown very good docking score against Candida albicans. Figure-1(A and B), represents the docked view of all the synthesized compounds at the active site of the enzyme PDB ID 1AI9.

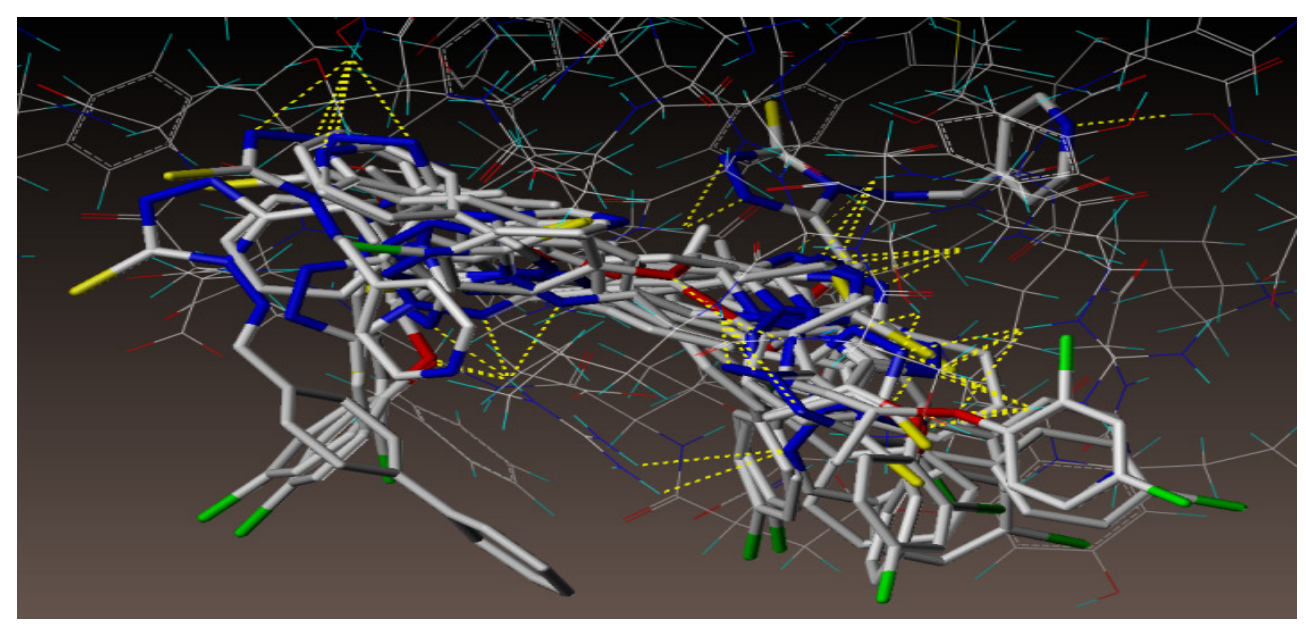

A

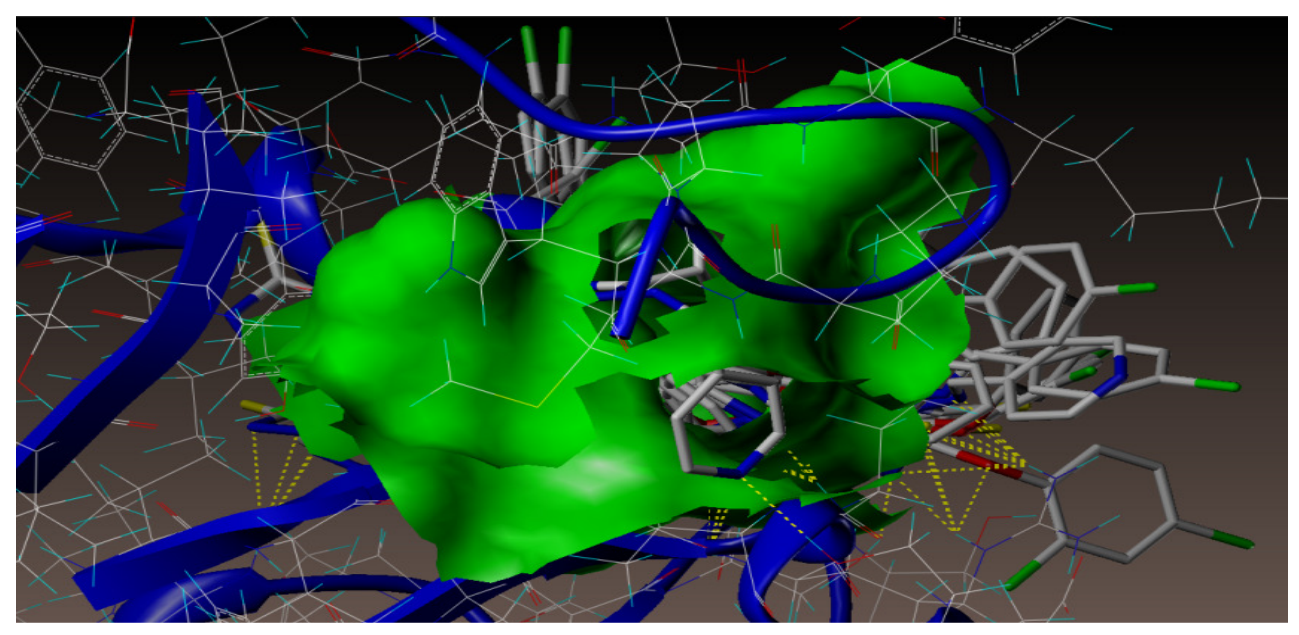

B

Fig.-1: Docked View of all the Compounds at the Active Site of the Enzyme PDB ID: 1AI9

As depicted in the Fig.-2(A-C), compound $\mathbf{6 k}$ has three hydrogen bonding interactions at the active site of the enzyme (PDB ID: 1AI9), among those three interaction, nitrogen atom present at $1^{\text {st }}$ position of triazole ring interacted with hydrogen of ARG56 (N-----H-ARG56; $2.76 \AA$ ), and nitrogen atom present at the $2^{\text {nd }}$ position of triazole ring has hydrogen bonding interaction with hydrogen of LYS57 (N------HLYS57; $2.44 \AA$ ) and remaining hydrogen bonding interaction is found to be raised from the nitrogen atom present at 4-chlorobenzylidene group with hydrogen of ARG79 (CH=N------H-ARG79; $2.40 \AA)$. 
RASĀYAN J. Chem.

Vol. 12 | No. 3 |1405 - 1417| July - September | 2019

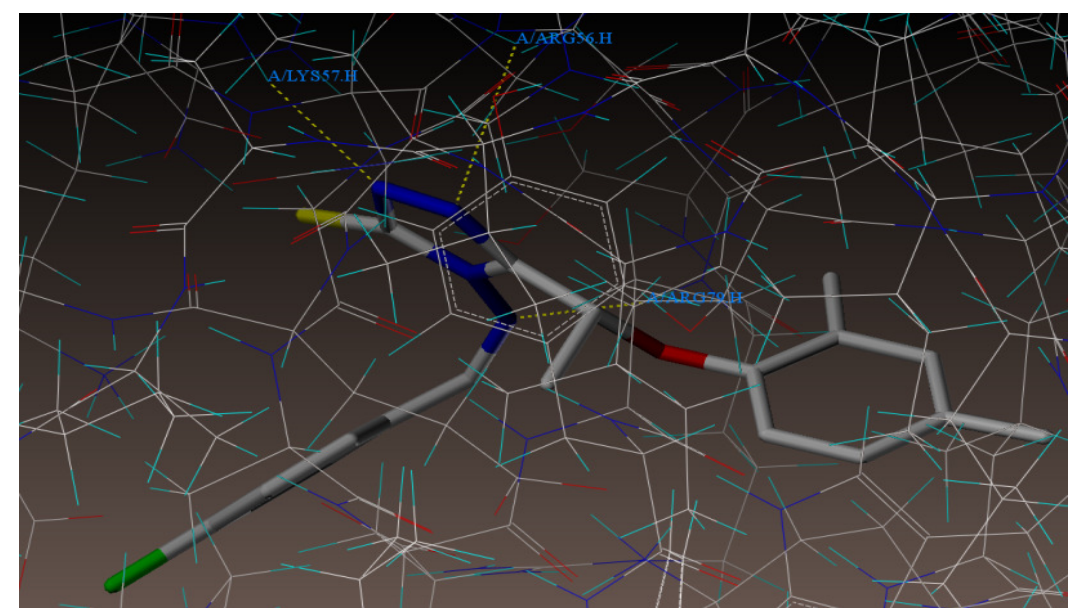

A

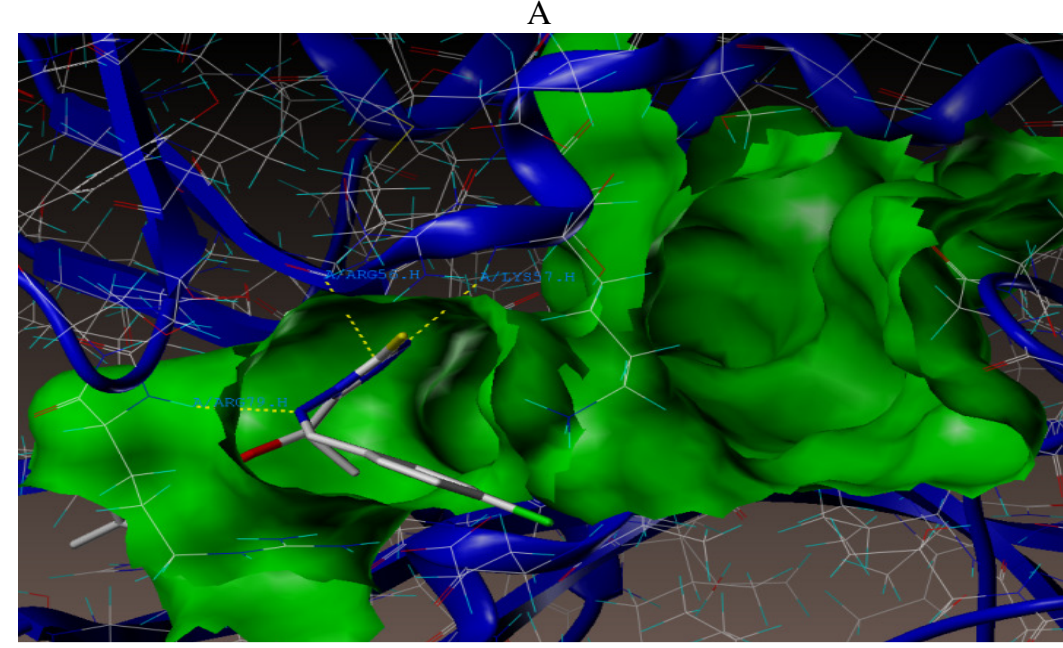

$\mathrm{B}$

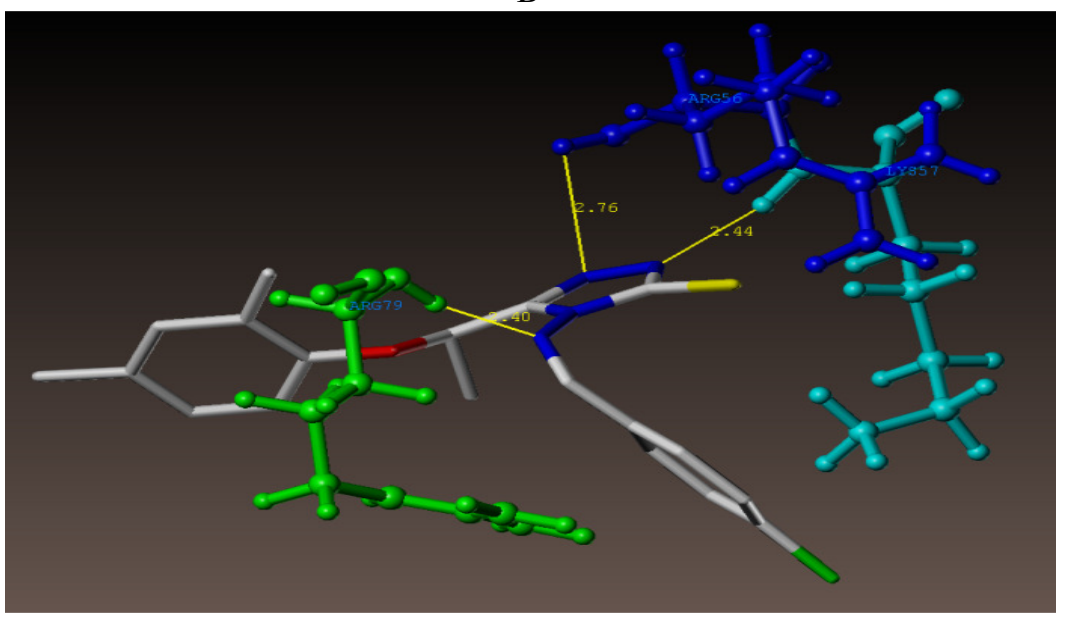

$\mathrm{C}$

Fig.-2: Docked View of Compound 6k at the Active Site of the Enzyme PDB: 1AI9

As depicted in Fig.-3(A-C), compound 6a, has four hydrogen-bonding interactions at the active site of the enzyme (PDB ID: 1AI9), among that four interaction, all the three interactions are from nitrogen atom present on the $1^{\text {st }}$ position of triazole ring with hydrogen atoms of ARG56 and LYS57 (N------H-ARG56; $2.47 \AA ; 2.84 \AA ; 2.48 \AA$ ) and remaining one hydrogen bonding interaction raised from the oxygen atom of phenoxy ethyl ring with hydrogen of ARG56 (O------H-ARG56; $2.07 \AA$ ). 
RASĀYAN J. Chem.

Vol. 12 | No. 3 |1405 - 1417| July - September | 2019

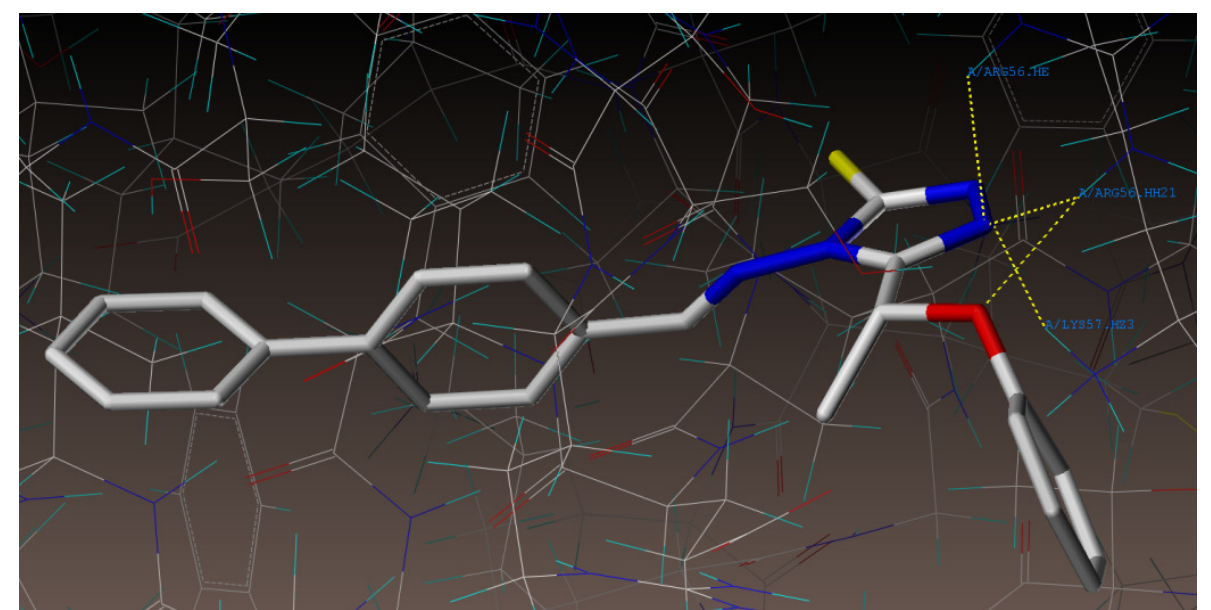

A

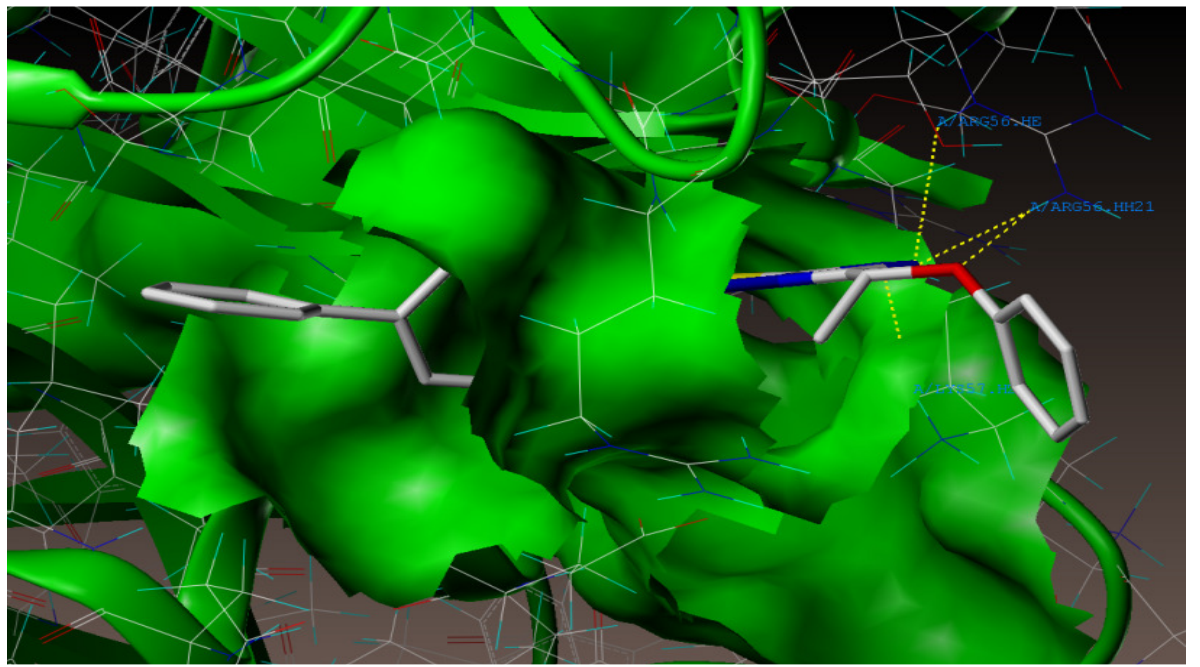

$\mathrm{B}$

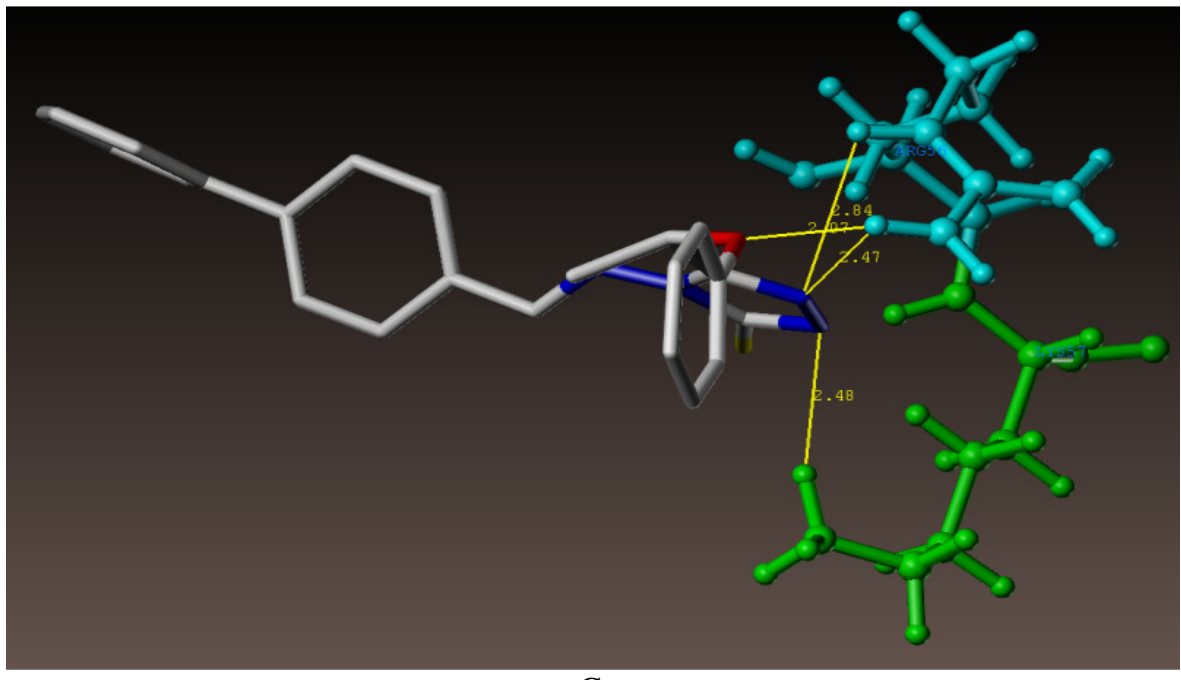

$\mathrm{C}$

Fig.-3: Interaction of Compound 6a at the Binding Site of the Enzyme (PDB ID: 1AI9)

As depicted in Fig.-4(A-C), flucanazole, makes four hydrogen bonding interaction at the active site of the enzyme (PDB ID: 1AI9) and all the four hydrogen bonding interaction are due to nitrogen atoms of traizole ring with hydrogen atom of amino acid residues SER78, LYS57 \& ARG56 (-N ---- H-SER78; HLYS57 \& H-ARG56).. 
RASĀYAN J. Chem.

Vol. 12 | No. 3 |1405 - 1417| July - September | 2019
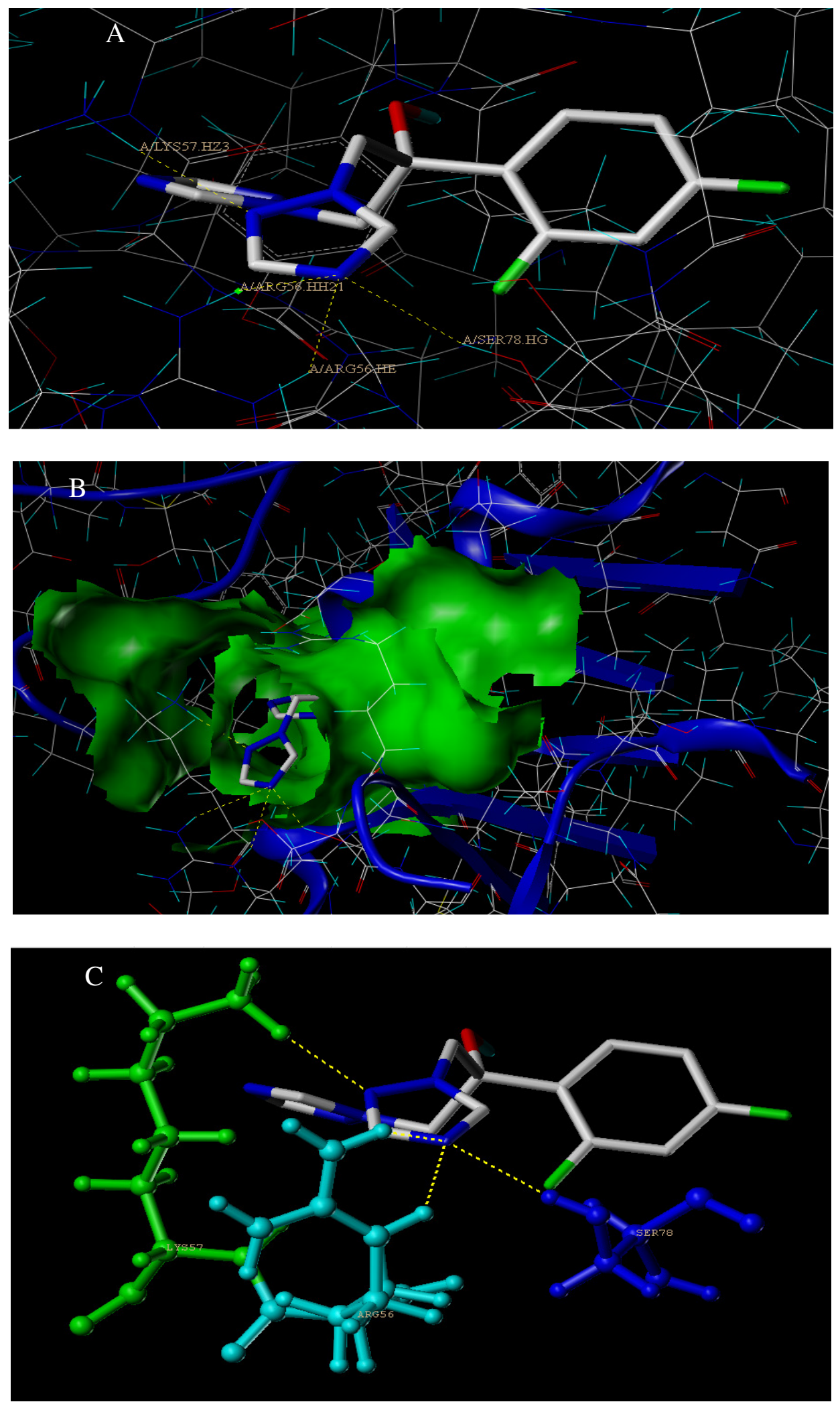

Fig.-4: Docked View of the Fluconazole at the Binding Site of the Enzyme PDB ID: 1AI9

In the docking study, the compounds have shown same type of interaction with amino acid residue as that of Fluconazole standard drug. The compounds have the same H-bonding interaction with amino acids LYS57 and ARG56 like that of Fluconazole. 
Thus, from these studies, we can corroborate the experimental findings, which suggest that triazole derivatives may act by inhibiting the dihydrofolate reductase enzyme.

Figure-5(A and B) represents the hydrophobic and hydrophilic amino acids surrounded to the studied compounds $6 \mathbf{k}$ and $\mathbf{6 a}$.

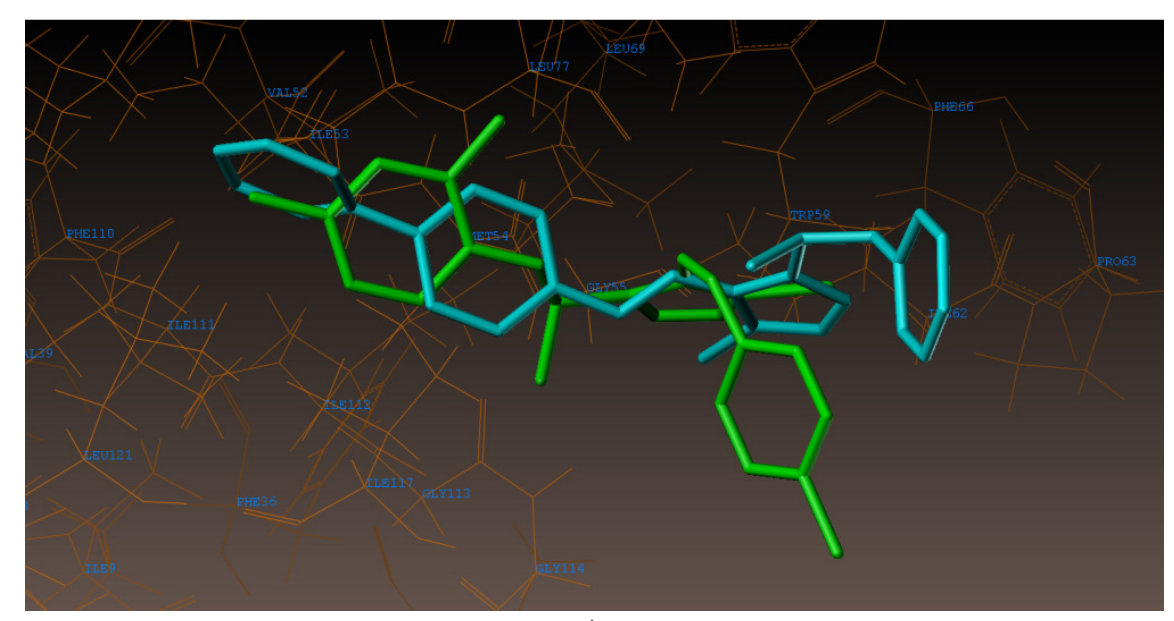

A

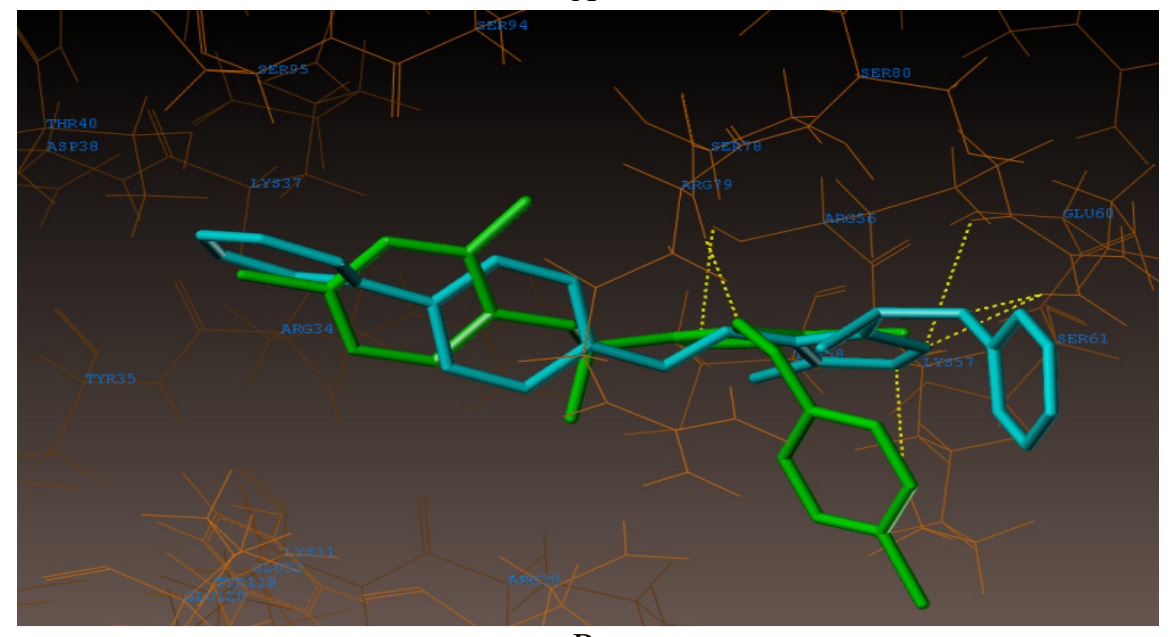

B

Fig.-5: (A) Hydrophobic amino acids surrounded to compounds 6k (green colour) and 6a (cyan colour). (B) Hydrophilic amino acids surrounded to compounds $\mathbf{6 k}$ and $\mathbf{6 a}$.

\section{CONCLUSION}

This study reports the successful synthesis of the title compounds. Out of the sixteen compounds hereby reported compound $\mathbf{6 a}, \mathbf{6 b}, \mathbf{6 c}, \mathbf{6 e}, \mathbf{6 f}, \mathbf{6 g} \mathbf{6 i}, \mathbf{6 k}, \mathbf{6 1 , 6 \mathrm { m }}$ showed the best activity against microbial strains Candida albicans with an MIC of $3.12 \mathrm{mg} / \mathrm{ml}$. The activity is found to be better than standard, we can corroborate the experimental findings, which suggest that triazole derivatives may act by inhibiting the dihydrofolate reductase enzyme.

\section{REFERENCES}

1. F. C. Odds, A. J P. Brown, N. A. R. Gow, Trends Microbiol., 11, 272(2003), DOI: 10.1016/S0966842X(03)00117-3.

2. D. K. Dalvie, A. S. Kalgutkar, S. C. Khojasteh-Bakht, R. S. Obach, J. P. O'Donnell, Chem. Res. Toxicol., 15, 269(2002), DOI:10.1021/tx015574b

3. W. S. Horne, M. K. Yadav, C. D. Stout, M. R Ghadiri, J. Am. Chem. Soc., 126, 15366(2004), DOI: $10.1021 / \mathrm{ja} 0450408$ 
RASĀYAN J. Chem.

Vol. 12 | No. 3 |1405 - 1417| July - September | 2019

4. M. B. Edmond, S. E. Wallace, D. K. McClish, M. A. Pfaller, R. N. Jones, R. P. Wenzel, Clin. Infect. Dis., 29, 239(1999), DOI: $10.1086 / 520192$

5. M. M. Roden, T. E. Zaoutis, W. L. Buchanan, T. A. Knudsen, T. A. Sarkisova, R. L. Schaufele, M. Sein, T. Sein, C. C. Chiou, J. H. Chu, D. P. Kontoyiannis, T. Walsh, J. Clin. Infect. Dis., 41, 634(2005), DOI: $10.1086 / 432579$.

6. K. E. Akri, K. Bougrin, J. Balzarini,; A. Faraj, R. Benhida, Bioorg. Med. Chem. Lett., 17, 6656 (2007), DOI:10.1016/j.bmcl.2007.08.077.

7. M. S. Karthikeyan, B. S. Holla, N. S. Kumari, Eur. J. Med. Chem., 43, 309(2008), DOI:10.1016/j.ejmech.2007.03.024.

8. N. S. Vatmurge, B. G. Hazra, V. S. Pore, F. Shirazi, P. S. Chavan, M. Deshpande, V. Bioorg. Med. Chem. Lett., 18, 2043(2008), DOI:10.1016/j.bmcl.2008.01.102

9. J. Zhang, H. Zhang, W. Cai, L. Yu, X. Zhen, A. Zhang, Bioorg. Med. Chem., 17, 4873(2009), DOI:10.1016/j.bmc.2009.06.019.

10. R. Jagasia, J. M. Holub, M. Bollinger, K. Kirshenbaum, M. G. Finn, J. Org. Chem., 74, 2964(2009), DOI: $10.1021 /$ jo802097m

11. D. Huber, H. Hübner, P. Gmeiner, J. Med. Chem., 52, 6860(2009), DOI: 10.1021/jm901120h.

12. E. D. Chrysina, É. Bokor, K.-M. Alexacou, M.-D. Charavgi, G. N. Oikonomakos, S. E. Zographos, D. D. Leonidas, N. G. Oikonomakos, L. Somsák, Tetrahedron: Asymmetry, 20,733(2009), DOI: 10.1016/j.tetasy.2009.03.021

13. A. Espinel-Ingroff, K. Boyle, D. Sheehan, J. Mycopathologia, 150, 101(2001), DOI:10.1023/A:1010954803886.

14. M.M. Pearson, P.D. Rogers, J.D. Cleary, S.W. Chapman, Ann. Pharmacother., 37, 420(2001), DOI:10.1345/aph.1C261.

15. E. K. Manavathu, J. L. Cutright, P.H. Chandrasekar, Antimicrob. Agents Chemother., 42, 3018(1998),

16. E.M. Johnson, A. Szekely, D.W. Warnock, J. Antimicrob. Chemother., 42, 741(1998), DOI: $10.1093 / \mathrm{jac} / 42.6 .741$.

17. J.C. Fung-Tomc, T.C. White, B. Minassian, E. Huczko, D.P. Bonner, Diagn. Microbiol. Infect. Dis. 35, 163(1999), DOI:10.1016/S0732-8893(99)00063-2.

18. D.S. Burgess, R.W. Hastings, Diagn. Microbiol. Infect. Dis., 38, 87(2000)

19. H. Goossens, Chemotherapy 51, 177(2005), DOI: 10.1016/S0732-8893(00)00173-5.

20. J. B. Bremner, J. I. Ambrus, S. Samosorn, Curr. Med. Chem., 14, 1459(2007), DOI: $10.2174 / 092986707780831168$.

21. C. Hubschwerlen, J.-L. Specklin, C. Sigwalt, S. Schroeder, H. H. Locher, Bioorg. Med. Chem. 11, 2313-2319. (2003), DOI:10.1016/S0968-0896(03)00083-X.

22. T. L. Gilchrist, Heterocyclic Chemistry, 2nd ed., Wiley: New York, (1992)

23. B. S. Holla, R. Gonsalves, S. Shenoy, Farmaco., 53, 574(1998), DOI:10.1016/S0014827X(98)00068-8.

24. B. S. Holla, B.Veerendra, M. K. Shivananda, N. S.Kumari, , Indian J. Chem., 42, 2010(2003)

25. M. Ashok, B. S. Holla, J. Pharmacol. Toxicol., 2, 256(2007)

26. D. J. Prasad, M. Ashok, P. Karegoudar, B. Poojary, B. S. Holla, N. S. Kumari, Eur. J. Med. Chem. 44, 551(2009), DOI:10.1016/j.ejmech.2008.03.025.

27. G. Turan-Zitouni, Z. A. Kaplancikli, M. T. Yildiz, P. Chevallet, D. Kaya, Eur. J. Med. Chem. 40, 607( 2005), DOI:10.1016/j.ejmech.2005.01.007.

28. K. Walczak, A. Gondela, Suwiński, J. Eur. J. Med. Chem., 39, 849(2004), DOI:10.1016/j.ejmech.2004.06.014.

29. B. S. Holla, K. N. Poojary, B. S. Rao, M. K. Shivananda, Eur. J. Med. Chem., 37, 511(2002), DOI:10.1016/S0223-5234(02)01358-2.

30. B. S. Holla, B. Veerendra, M. K. Shivananda, B. Poojary, Eur. J. Med. Chem., 38, 759(2003), DOI:10.1016/S0223-5234(03)00128-4.

31. M. Amir, K. Shikha, Eur. J. Med. Chem., 39, 535(2004) 
RASĀYAN J. Chem.

Vol. 12 | No. 3 |1405 - 1417| July - September | 2019

32. A. Almasirad, S. A. Tabatabai, M. Faizi, , A. Kebriaeezadeh, N. Mehrabi, A. Dalvandi, A. Shafiee, Bioorg. Med. Chem. Lett., 14, 6057(2004), DOI:10.1016/j.bmcl.2004.09.072.

33. K. Masuda, T. Toga, N. Hayashi, J. Labelled Compd., 11, 301(1975), DOI:10.1002/jlcr.2590110219.

34. E. Schreier, Helv. Chim. Acta, 59, 585(1976), DOI:10.1002/hlca.19760590223.

35. S. Budavari, Ed. The Merck Index, 12th ed.; Merck Co. Inc: White House Station, NJ, (1996)

36. J. Haber, Cas. Lek. Cesk., 140, 596(2001)

37. J. Gasteiger, M. Marsili, Tetrahedron, 36, 3219(1980).

38. Tripos International (2012) Sybyl-X 2.0, Tripos International, St. Louis, MO, USA.

[RJC-5200/2019] 